Revista de Matemática: Teoría y Aplicaciones 2000 7(1-2) : 117-124

CIMPA - UCR - CCSS ISSN: 1409-2433

\title{
UN PROBLEMA DE LOCALIZACIÓN DE PLANTAS DE GRAN ESCALA
}

\author{
Miguel Angel Gutiérrez Andrade* - Sergio De los Cobos Silva** \\ Blanca Rosa PÉrez Salvador $\stackrel{* * *}{-}$ John Goddard ${ }^{* * * *}$
}

Recibido: 16 Junio 2000

\begin{abstract}
Resumen
En este artículo se desarrolla un algoritmo heurístico y su correspondiente implementación para resolver un problema de localización de plantas de gran escala, en donde surgen potencialmente más de 640 plantas a localizar a lo largo de la República Mexicana. Originalmente se trató de obtener solución exacta al problema, usando dos técnicas clásicas: descomposición de Benders y ramificación y acotamiento. Ambas técnicas resultan adecuadas y eficientes para resolver problemas de tamaño chico, pero las implantaciones en computadora para este problema no convergieron después de muchas horas de proceso. Se requería obtener una solución al problema mediante alguna técnica que quizá no diera la solución exacta, pero sí una solución de buena calidad. Para la solución de este problema real, se empleó la técnica de recocido simulado con excelentes resultados.
\end{abstract}

Palabras clave: localización, sobrecalentamiento simulado, recocido simulado, heurística.

\begin{abstract}
We develop an heuristic algorithm and its implementation for solving a large scale facility location problem, where there can arise over 640 facilities to be located in Mexico. Originally, we tried to obtain an exact solution to the problem, using two classical techniques: Benders decomposition, and branch and bound. Both techniques

\footnotetext{
${ }^{*}$ Depto. de Sistemas, Universidad Autónoma Metropolitana-Azcapotzalco, Av. San Pablo 180, Colonia Reynosa Tamaulipas, 02200 México, D.F. México; E-Mail: gama@hp9000a1.uam.mx

${ }^{* *}$ Depto. de Ingeniería Eléctrica, Universidad Autónoma Metropolitana-Iztapalapa, Av. Michoacán y La Purísima, Col. Vicentina, CP 09340 México D.F., México; E-Mail: cobos@xanum.uam.mx

*** Depto. de Matemáticas, Universidad Autónoma Metropolitana-Iztapalapa, Av. Michoacán y La Purísima, Col. Vicentina, CP 09340 México D.F., México.

${ }^{* * * *}$ Misma dirección que S. de los Cobos; E-Mail: jgc@xanum.uam.mx
} 
are adequate and efficient for solving low-scale problems, but computer implementations for this problem did not converge after several hours of computing. Hence, we needed a good solution even if it was not exact. We used the simulated annealing technique with excellent results.

Keywords: facility location, simulated annealing, heuristics.

\section{Mathematics Subject Classification: 90B 80}

\section{Descripción del problema de localización de plantas}

El problema de localización de plantas tiene la siguiente estructura: hay $n$ sitios en una región que requiere un producto. La demanda para el producto en el sitio $i$ es $d_{i}$ unidades, para $i=1, \ldots, n$. La demanda tiene que satisfacerse manufacturando el producto dentro de la región. Se necesitan $m$ o menos plantas para manufacturar el producto que satisfaga la demanda, donde $m$ se especifica. El costo por construir una planta en el sitio $i$ es $f_{i}$ unidades monetarias. Si una planta se construye en el sitio $i, k_{i}$ unidades es la capacidad de producción.

Si existe una ruta de transporte entre el sitio $i$ al $j, k_{i j}$ es la capacidad sobre el horizonte de planeación y $c_{i j}$ es el costo de transporte de una unidad entre el sitio $i$ al $j$ y $f_{i j}$ es el costo fijo por usar la ruta entre el sitio $i$ al $j$. El problema es determinar un subconjunto óptimo de sitios de localización de las plantas y un plan de transporte que minimice el costo total de construcción de plantas y el transporte de productos. El problema de determinar un subconjunto de sitios para localizar plantas y los costos fijos de transporte entre sitios es un problema de optimización combinatoria. Una vez que se conoce una solución de este problema, es decir, se ha decidido donde colocar las plantas, el problema de determinar la cantidad óptima transportada a lo largo de las rutas es un simple problema de transporte. Así, cada vez que se asignan las plantas a sitios, se debe resolver un problema de transporte para obtener la distribución óptima del producto.

Este problema puede formularse como un problema entero usando variables de decisión con las siguientes interpretaciones:

$y_{i}=1 \quad$ si la planta se localiza en el sitio $i$,

$y_{i}=0 \quad$ en otro caso.

$y_{i j}=1 \quad$ si existe una ruta de transporte del sitio $i$ al $j$,

$y_{i j}=0 \quad$ en otro caso.

$x_{i j}=$ cantidad (en unidades) del producto transportado del sitio $i$ al $j$.

Entonces el problema de localización de plantas puede formularse matemáticamente como:

$$
\begin{aligned}
\min \sum_{i} \sum_{j} c_{i j} x_{i j}+\sum_{i} f_{i} y_{i} & +\sum_{i} \sum_{j} f_{i j} y_{i j} \\
\text { Sujeto a } \quad \sum_{j} x_{i j}-k_{i} y_{i} & \leq 0 \quad \forall i \\
x_{i j}-k_{i j} y_{i j} & \leq 0 \quad \forall i, j \\
\sum_{i} x_{i j} & \geq d_{j} \quad \forall j \\
\sum_{i} y_{i} & \leq m \\
x_{i j} \geq 0, \forall i, j ; y_{i}, y_{i j} & =0 \text { ó } 1 \quad \forall i, j
\end{aligned}
$$


El problema de localización de plantas está clasificado dentro de la familia $N P$-completa y por lo tanto los algoritmos conocidos para resolver el problema de manera exacta, son no polinomiales, como por ejemplo, enumeración implícita y ramificación y acotamiento.

\section{Aplicación del recocido simulado}

El algoritmo de recocido simulado puede usarse para atacar este problema y obtener soluciones que no necesariamente son óptimas, pero se puede mostrar un comportamiento muy favorable del método con respecto a instancias que se conoce la solución. Para mayores detalles acerca de la filosofía del algoritmo vea [1].

\subsection{Descripción del algoritmo}

El algoritmo de recocido simulado se implementó de la siguiente manera:

- El espacio de soluciones $S$, para el problema combinatorio, está representado por todos los vectores binarios $y_{1}, y_{2}, \ldots, y_{n}$ tal que $y_{i}=0$ ó 1 y $\sum_{i=1}^{n} y_{i} \leq m$. Donde $y_{i}=0$ si no se coloca planta en el sitio $i$ y $y_{i}=1$ si se coloca planta en el sitio $i$.

- La función de costo a minimizar, se escoge como: $f=\sum_{i} \sum_{j} c_{i j} x_{i j}+\sum_{i} f_{i} y_{i}$ donde $x_{i j}$ es la cantidad (en unidades) del producto transportado del sitio $i$ al sitio $j$ y $f$ es la suma del costo de la localización de las plantas, más el costo de transporte del producto. Para este caso los costos fijos $f_{i j}$ se tomaron iguales a cero ya que no existe ningún costo fijo por el uso de los caminos.

- Las nuevas soluciones se obtienen a partir de dos mecanismos de generación: uno que selecciona aleatoriamente un sitio $i$, si existe planta en el sitio $i$ se quita y si no existe, entonces se coloca una planta. El otro mecanismo genera aleatoriamente un sitio $i$, si existe una planta en $i$ se cambia a otro sitio $j$ donde no exista, tomado de manera aleatoria y si no existe planta en el sitio $i$, se busca aleatoriamente un sitio $j$ donde exista una planta y se intercambia. La selección de uno u otro mecanismo se hace de manera aleatoria. Con probabilidad $p$ se hace intercambio de plantas y con probabilidad $1-p$ se abre una planta o se cierra. El valor de $p$ puede variarse dependiendo del parámetro de control.

- La actualización de la función de costo entre la solución actual y la nueva solución puede hacerse fácilmente en la parte de costos fijos únicamente al quitar, agregar o intercambiar los costos de plantas que se den de alta y de baja. La parte del costo de transporte debe calcularse para cada nueva configuración usando la solución actual y modificando los suministros variados por medio de penalizaciones.

- El valor inicial $c$ del parámetro de control se obtuvo de manera que la tasa de aceptación de las soluciones propuestas fuera mayor o igual al $95 \%$.

- Los valores subsiguientes del parámetro de control se obtuvieron decrementando éste en $1 \%$ donde no se aceptó ninguna nueva solución después de explorar $r$ veces sobre las soluciones vecinas. 
- El número de iteraciones $r$ para un valor fijo del parámetro de control se tomó igual al tamaño máximo de la vecindad de una solución $n+n(n-1)=n^{2}$

La Figura 1 describe el algoritmo usado de recocido simulado para este problema.

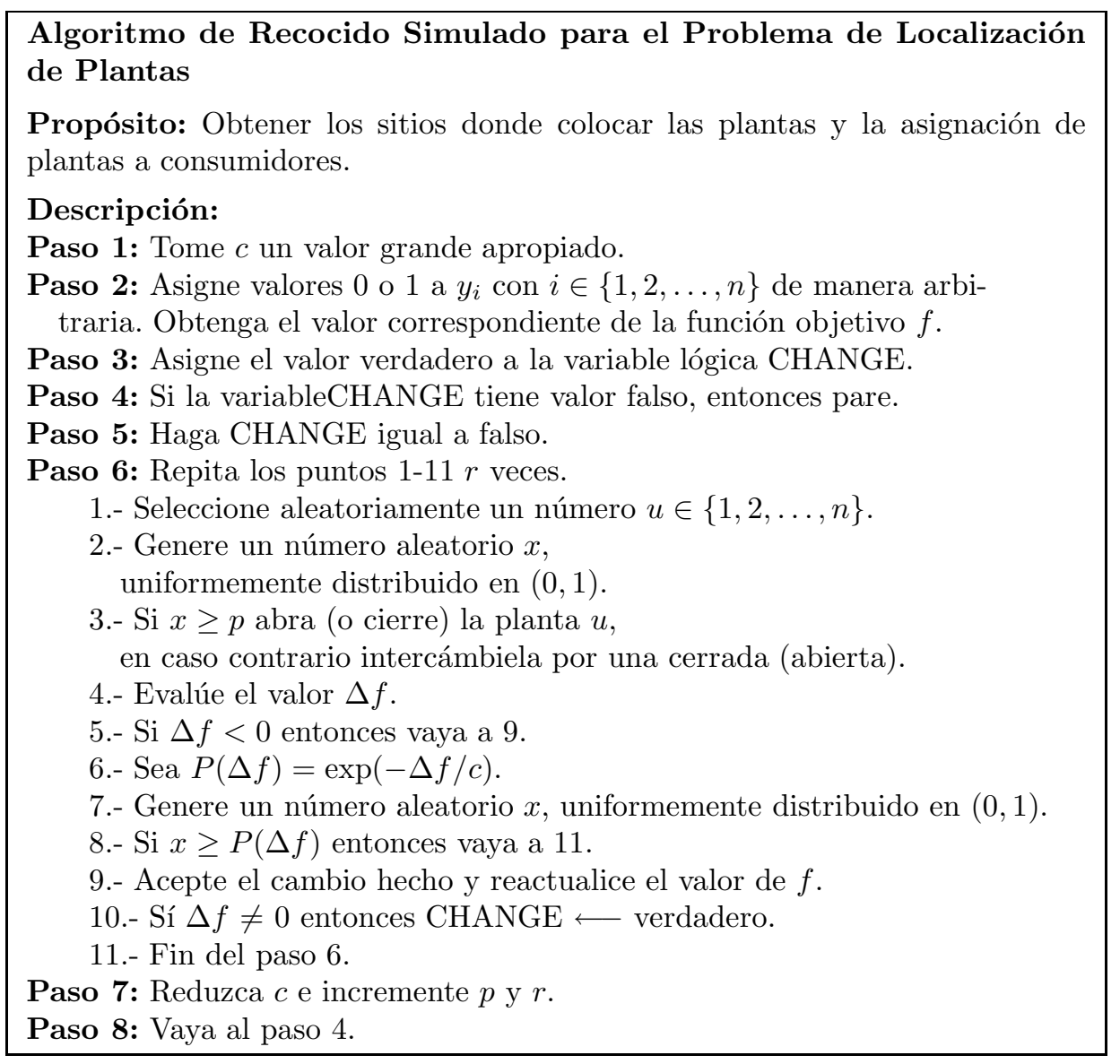

Figura 1: Algoritmo de recocido simulado para el problema de localización de plantas

\subsection{Experiencia computacional}

La prueba del algoritmo se llevó a cabo generando de manera aleatoria instancias del problema de localización de plantas. Para valores preestablecidos del número $m$ de plantas y el número $n$ de centros de consumo, se generaron 100 instancias aleatorias del problema. Para cada planta se tomó como costo fijo un número aleatorio con distribución uniforme entre (100-500); para cada consumidor se tomó como costo de transporte un número aleatorio con distribución uniforme entre (40-200) y finalmente para la demanda en cada sitio, se tomó un número aleatorio con distribución uniforme entre (10-100).

Para calcular la eficiencia del algoritmo se usó el siguiente índice

$$
\text { eficiencia }=1-\frac{f-f_{o p t}}{f_{o p t}}
$$


donde $f$ denota el valor de la función objetivo en la solución obtenida y $f_{\text {opt }}$ es el valor óptimo de la instancia del problema, donde el valor $f_{\text {opt }}$ se obtiene usando un algoritmo exacto. Para obtener los óptimos que se requieren en la Tabla 1 se usó el algoritmo de Khumawala [3].

La Tabla 1 reporta la eficiencia del algoritmo con respecto al valor óptimo de la función objetivo. Los valores que aparecen son con base en los promedios obtenidos en las 100 instancias resueltas para cada valor del número $m$ de plantas y del número $n$ de sitios de consumo.

\begin{tabular}{|c|rrrr|}
\hline $\begin{array}{c}\text { Número } \\
\text { de Nodos }(n)\end{array}$ & \multicolumn{4}{|c|}{ Número de Plantas $(m)$} \\
\hline & 5 & 10 & 15 & 20 \\
5 & 0.9899 & 0.9966 & 0.9989 & 0.9977 \\
\hline & 10 & 15 & 25 & 30 \\
10 & 0.9962 & 0.9929 & 0.9957 & 0.9912 \\
\hline & 15 & 25 & 35 & 40 \\
15 & 0.9924 & 0.9985 & 0.9963 & 0.9796 \\
\hline & 20 & 25 & 35 & 40 \\
20 & 0.9919 & 0.9847 & 0.9863 & 0.9895 \\
\hline & 25 & 30 & 35 & 40 \\
25 & 0.9911 & 0.9884 & 0.9943 & 0.9886 \\
\hline & 30 & 40 & 50 & 60 \\
30 & 0.9891 & 0.9920 & 0.9881 & 0.9866 \\
\hline & 35 & 40 & 50 & 60 \\
35 & 0.9904 & 0.9906 & 0.9898 & 0.9923 \\
\hline & 40 & 45 & 50 & 60 \\
40 & 0.9857 & 0.9943 & 0.9917 & 0.9862 \\
\hline
\end{tabular}

Cuadro 1: Eficiencia promedio del algoritmo para las 100 instancias.

\section{Problema de Localización de Plantas de Gran Escala}

El problema consiste en la localización de plantas productoras de tortilla empacada y tiene la siguiente estructura: se consideran 80 centros de demanda en la República Mexicana que corresponden a 85 ciudades con más de 50,000 habitantes que requieren del producto. Se tiene que satisfacer la demanda, para lo anterior se requiere construir plantas de tortilla empacada en algunas zonas. El costo por construir una planta en un sitio, depende del tamaño de la planta (para propósitos de las corridas del modelo se consideraron tres tamaños: 2 toneladas diarias, 5.34 toneladas diarias, 16 toneladas diarias). Además se consideró que el costo de los insumos (harina de maíz) depende de la distancia de la planta a la fábrica de harina más cercana a la misma.

El problema consiste en determinar un conjunto óptimo de sitios de localización de las plantas, número de plantas y sus respectivas capacidades; así como la zona de influencia 
de las mismas. Las zonas de influencia se reflejan en un plan óptimo de transporte en el horizonte de planeación (a 10 años).

Se desea minimizar el costo total de construcción anualizado de las plantas y el costo total de transporte de la tortilla de maíz empacada.

Entre los supuestos usados tenemos:

Costos. Como ya se mencionó, existen dos componentes principales en los costos del problema a minimizar: Costos de transporte y costos fijos.

Costos de transporte. Para obtener los costos de transporte se consideraron las distancias existentes en la red carretera entre cada par de sitios. Estas distancias se obtuvieron del Atlas de Carreteras de México editado por Guía Roji (1999) en donde se tomaron en cuenta las rutas comerciales. Cabe mencionar que existen dos rutas marítimas que son: Mazatlán- La Paz y Mochis-La Paz, en donde se tomó un costo de transporte del doble del considerado por vía terrestre. El costo de transporte se obtiene multiplicando la distancia por el costo por kilómetro-tonelada transportada. El valor del costo por kilómetro por tonelada transportada se consideró homogéneo en todo el territorio e igual a 1.25 pesos.

Costos fijos. Los costos fijos consisten de varios rubros: Costos de inversión (costo de depreciación de equipo), costos de insumos, costos de operación, costos de distribución, gastos financieros, utilidad y varios. Todos los costos fijos se tomaron anualizados.

Demandas. Con base en las estimaciones de consumo potencial de tortilla y de la distribución del mismo por entidad federativa y ciudad, se obtuvo una demanda potencial en cada uno de los sitios considerados. Se tomaron en cuenta dos escenarios de acuerdo a que el consumo de la tortilla empacada, en principio, se limita únicamente a las clases media y alta. Uno de los escenarios supone la captación de un $10 \%$ de demanda y el otro del $20 \%$.

Número de plantas. Debido a la gran diversidad en cuanto al tamaño de la demanda, se dividieron los centros de demanda en dos clases: (1) Zona Metropolitana, Guadalajara, Monterrey y Puebla. (2) los 76 centros restantes.

Para la primera clase, es claro que en la solución óptima se deben colocar plantas de al menos el tamaño de la demanda en las mismas y de capacidad máxima además de dejar la opción al modelo de colocar plantas adicionales para satisfacer la demanda de sitios cercanos a la misma. En la segunda clase se tomaron como posibles opciones de solución, la construcción de dos plantas de 2 toneladas diarias, 2 plantas de 5.34 toneladas diarias, 4 plantas de 16 toneladas diarias; dando un total de ocho posibilidades. Esto es, la solución puede ser una combinación de las ocho posibilidades.

Centros de insumo. Se consideraron 19 puntos de insumos de harina de maíz, estos lugares corresponden a la existencia de fábricas de harina de maíz ocho de MICONSA (localizadas en Los Mochis, Sin.; Guadalajara, Jal.; Tlalnepantla, Edo. de Mex.; Arriaga, Chis.; Jaltipán, Ver.; Atlacomulco, Edo. de Mex.; Acapulco, Gro. y Monterrey, N.L.) y once de MASECA (localizadas en Monterrey, N.L.; Río Bravo, Tamps.; Tampico, Tamps.; Chinameca, Ver.; Teotihuacan, Edo. de Mex.; Zamora, Mich.; Guadalajara, Jal.; Acaponeta, Nay.; Culiacán, Sin.; Obregón, Son. y Chihuahua, Chi.) Para cada posible lugar de localización se considera la distancia mínima entre dicho lugar y todos los puntos de insumo. 


\subsection{Enfoques clásicos de solución}

El problema se trató de solucionar de manera exacta mediante dos técnicas clásicas que se han venido utilizando: descomposición de Benders [6] y ramificación y acotamiento [3] y [5]. Ambas técnicas resultan adecuadas y eficientes para resolver problemas de tamaño chico, pero para el problema anteriormente descrito, donde se tienen 640 variables binarias estas técnicas son impracticables, pues para instancias mayores de 60 variables binarias presentan problemas de tiempo de ejecución excesivo.

\subsection{Solución con recocido simulado}

Como ya se comentó el algoritmo de recocido simulado puede usarse para resolver el problema expuesto. Para ello se procedió a implantar un código de computadora que resolviera el problema. El lenguaje que se usó en la programación fue FORTRAN 77; el programa consta de alrededor de 1800 líneas de código y corre en una computadora PC. En este programa se especificó un mecanismo de generación de vecindades y un programa de enfriamiento. El mecanismo de generación de vecindades y el programa de enfriamiento ya se especificaron en la Sección 2. Se hicieron varias corridas para cada combinación del periodo y reducción del parámetro de control.

\subsection{Resultados obtenidos}

En ambas corridas (10\% de tortillas empacadas tienen distancias pequeñas. Esto es, sólo existe transferencia entre ciudades cercanas lo que asegura el plan de distribución óptimo. Existen pocos movimientos en la red carretera. Entre 140 ligas interciudades existentes, sólo la mitad (aproximadamente 70) de ellas tienen flujo de mercancía. Se observa que los costos de transporte (flete) son muy pequeños.

Dado que el costo de colocar una planta es muy superior al costo de transporte, optimizar las inversiones es predominante, entonces es lógico que la capacidad total debe ser ligeramente mayor que la demanda total. En las corridas, las capacidades instaladas son $0.44 \%$ únicamente $0.15 \%$ las capacidades de las plantas. En la mayoría de los casos, la capacidad instalada en cada ciudad es similar a la demanda de la misma, lo cual es razonable ya que se evitan transferencias de la mercancía entre las ciudades. Esto significa que el modelo de optimización tiende a satisfacer las demandas locales.

Para el caso de $10 \%$ de demanda, el costo de transporte es slo $1.25 \%$ de la inversin total.Para el caso de $20 \%$ de demanda, el costo de transporte es slo $0.82 \%$ de la inversin total. Las zonas de influencia se reducen a regiones pequeñas.

Existen varias ciudades que no requieren ninguna inversión ya que sus demandas son pequeñas y pueden satisfacerse por zonas aledañas. A pesar de considerar sólo el $10 \%$ demanda potencial, se requiere muchas plantas en que invertir, lo cual implica un mercado futuro atractivo. Se observa que el modelo introduce, por lo general plantas grandes para satisfacer las demandas, esto se debe a que los costos marginales son menores que los costos en que se incurriría si se instalaran plantas más chicas. El modelo únicamente introduce plantas chicas o medianas para ajustar la demanda a la oferta. Por otro lado, como el costo de inversión es muy superior al costo de transporte, es preferible instalar plantas 
grandes y hacer transferencias de flujo para satisfacer demandas en los centros donde la demanda es menor.

\section{Conclusiones}

En este artículo se presentó un algoritmo de recocido simulado para resolver el problema de localización de plantas. Este algoritmo se probó para instancias de tamaño medio, comparando su eficiencia con respecto a los valores óptimos dados por un algoritmo exacto. Se observó que la eficiencia mínima fue del 0.9796, por lo que concluimos que el algoritmo heurístico da soluciones de buena calidad.

Los algoritmos exactos que resuelven el problema de Localización de Plantas son viables para problemas en donde el número de plantas a localizar es pequeño o mediano, en este estudio, para 40 plantas ya los algoritmos exactos tienen problemas de convergencia, para 80 plantas los algoritmos exactos no convergieron.

El algoritmo de recocido simulado se usó para resolver un problema de Localización de Plantas de gran escala. Para este problema se requería localizar 640 plantas a lo largo de la República Mexicana. Los resultados obtenidos fueron excelentes.

Se menciona que los resultados del modelo fueron excelentes ya que en la solución del problema real se observa: pocos movimientos en la red carretera; las distancias recorridas entre una planta y un centro de demanda es pequeña, es decir, el modelo tiende a satisfacer demandas locales; la capacidad total instalada es ligeramente mayor a la utilizada, esto implica que se aprovecha casi totalmente las capacidades de las plantas

\section{Referencias}

[1] Aarts, E.; Korst, J. (1989) Simulated Annealing and Boltzmann Machines. John Wiley \& Sons, Chichester.

[2] Emden-Weinert, T., Proksch, M., (1999) "Practice simulated annealing for the airline crew scheduling problem", Journal of Heuristics 5(4): 419-436.

[3] Khumawala, B.M. (1972) "An efficient branch and bound algorithm for the warehouse location problem", Management Science 18: 718-731.

[4] Geraldo, R.; Mateus, G.R.; Luna, H.; Sirihal, A. (2000) "Heuristics for distribution network design in telecommunication", Journal of Heuristics 6(1): 133-150.

[5] Mirchandani, P.B.; Reilly, J.M. (1986) "Spatial nodes in discrete location problems", Annals of Operations Research 6: 203-222.

[6] Mirchandani, P.B.; Francis, R.L. (Eds.) (1990) Discrete Location Theory. John Wiley \& Sons, New York.

[7] Privault, C.; Herault, L. (1998) "Solving a real world assignment problem with a metaheuristic", Journal of Heuristics 4(4): 383-398. 